\title{
Incidence and factors associated with emergency department visits for recurrent skin and soft tissue infections in patients in California, 2005-2011
}

\author{
L. MAY ${ }^{1 *}$, E. Y. KLEIN ${ }^{2,3}$, E. M. MARTINEZ ${ }^{3}$, N. MOJICA ${ }^{3}$ AND L. G. MILLER ${ }^{4}$ \\ ${ }^{1}$ University of California-Davis, Department of Emergency Medicine, Sacramento, CA, USA \\ ${ }^{2}$ Johns Hopkins University, Department of Emergency Medicine, Baltimore, MD, USA \\ ${ }^{3}$ Center for Disease Dynamics, Economics \& Policy, Washington, DC, USA \\ ${ }^{4}$ Division of Infectious Diseases, Harbor-University of California, Los Angeles (UCLA) Medical Center, and \\ Los Angeles Biomedical Research Institute at Harbor-UCLA Medical Center, Torrance, CA, USA
}

Received 19 July 2016; Final revision 22 September 2016; Accepted 8 November 2016; first published online 5 December 2016

\section{SUMMARY}

More than 2 million visits for skin and soft tissue infections (SSTIs) are seen in US emergency departments (EDs) yearly. Up to $50 \%$ of patients with SSTIs, suffer from recurrences, but associated factors remain poorly understood. We performed a retrospective study of patients with primary diagnosis of SSTI between 2005 and 2011 using California ED discharge data from the State Emergency Department Databases and State Inpatient Databases. Using a multivariable logistic regression, we examined factors associated with a repeat SSTI ED visits up to 6 months after the initial SSTI. Among 197371 SSTIs, 16.3\% were associated with a recurrent ED visit. We found no trend in recurrent visits over time $\left(\chi^{2}\right.$ trend $\left.=0 \cdot 68, P=0 \cdot 4\right)$. Race/ethnicity, age, geographical location, household income, and comorbidities were all associated with recurrent visits. Recurrent ED visits were associated with drug/alcohol abuse or liver disease [odds ratio (OR) $1 \cdot 4,95 \%$ confidence interval (CI) $1 \cdot 3-1 \cdot 4]$, obesity (OR $1 \cdot 3,95 \%$ CI $1 \cdot 2-1 \cdot 4$ ), and in infections that were drained (OR $1 \cdot 1,95 \%$ CI $1 \cdot 1-1 \cdot 1)$ and inversely associated with hospitalization after initial ED visit (OR 0·4, 95\% CI 0·3-0.4). In conclusion, we found several patient-level factors associated with recurrent ED visits. Identification of these high-risk groups is critical for future ED-based interventions.

Key words: Skin and soft tissue.

\section{INTRODUCTION}

National trends in the United States have shown a marked increase in ambulatory visits and hospitalizations for skin and soft-tissue infections (SSTIs) coinciding with the emergence of community-acquired methicillinresistant Staphylococcus aureus (CA-MRSA), which is

\footnotetext{
* Author for correspondence: L. May, MD, MSPH, MSHS, Department of Emergency Medicine, University of California-Davis, 4150 V Street, Suite 2100, Sacramento, CA 95817, USA. (Email: 1smay@ucdavis.edu)
}

the primary cause of up to $64 \%$ of SSTIs seen in emergency departments (EDs) nationwide [1-4]. More than $90 \%$ of SSTIs are treated in the outpatient setting [5]. Among the estimated $34 \cdot 8$ million outpatient visits for SSTIs between 2005 and 2011, about $33 \%$ of these were seen in the ED [6-8].

Up to $70 \%$ of SSTI medical visits are complicated by recurrent infections [9-12]. In non-ED settings and small cohort studies, risk factors for SSTI recurrence include MRSA aetiology, recent hospitalization, recent SSTI, cephalosporin use, comorbidities (e.g. diabetes, obesity, HIV), and geographical location 
[12-16]. While there is a growing literature on predictors of recurrent CA-MRSA and S. aureus SSTIs, there are limited data on sociodemographic characteristics and overall predictors of recurrent SSTIs from large cohorts for those patients presenting to the ED for SSTI care.

Improved knowledge of SSTI recurrence epidemiology and risk factors in patients who present to ED settings is critical for informing treatment guidelines, and for guiding future study of ED-based interventions, particularly for high-risk patients. Such knowledge may assist clinicians identify patients at high risk for visits to the ED for recurrent infection. Here we describe rates and predictors of visits to the ED for recurrent SSTIs in a very large population of patients visiting the ED for an SSTI.

\section{MATERIALS AND METHODS}

\section{Study design, setting, and data collection}

We performed a retrospective study of recurrent SSTIs in patients who visited an ED in California between 2005 and 2011. California, with more than 37 million persons (in 2010), comprises over $12 \%$ of the US population, the highest population of any US state, with a large proportion of publicly and underinsured patients and higher than national proportion of patients that use the ED for care [17-19]. Besides the large size, we chose California because of the ability to track readmissions across facilities within the entire state, the amount of clinical information encoded in its health records, and because it was one of the earliest spots in the United States to be affected by the CA-MRSA epidemic [3, 20-22].

The study utilized California ED discharge data from the State Emergency Department Databases (SEDD) and the State Inpatient Databases (SID) from the Healthcare Cost and Utilization Project (HCUP) of the US Department of Health and Human Services Agency for Healthcare Research and Quality. The dataset included all ED visits and subsequent hospitalizations at non-federal (e.g. nonmilitary, Veterans Administration, or Indian Health Service), short-term general, and other speciality hospitals in California between 2005 and 2011

\section{Statistical methods}

We used multivariable logistic regression to investigate the magnitude to which sociodemographic factors and comorbidities at the time of hospitalization were associated with the odds of having one or more recurrent SSTI visits. Variables were selected based on the aetiology of SSTIs, comorbidities associated with an increased risk of SSTIs, and sociodemographic characteristics associated with higher risk of recurring SSTIs based on review of the prior literature $[10,12,13,23]$. We examined the correlation between variables using Spearman's correlation coefficient, and we assessed multicollinearity using the variance inflation factor. Additionally, a proportional trend analysis was conducted to determine if the rate of SSTI recurrent visits changed over time. Stata v. 14.1 (StataCorp, USA) was used for all analyses.

The analysis included all patients with a principal diagnosis of uncomplicated SSTI or 'other skin and subcutaneous infections', using previously described definitions [5]. The included ICD-9-CM codes are listed in Table 1. A recurrent visit was defined as any patient who returned to the ED for an SSTI between 2 weeks and 6 months (180 days) after an initial visit. This conservative time-frame allowed a focus on recurrent cases proximal to the initial SSTI while excluding follow-up visits that were likely for the same infection.

Data on primary visits were included for years 2006-2010. Primary visit data from years 2005 and 2011 were excluded from the final analysis because recurrent visits within 180 days were not accurately represented for patients whose first visit may have fallen before January 2005 or whose recurrent visit may have fallen after December 2011. Data from 2005 were used only to determine if a primary visit in 2006 was indeed a primary visit and not a recurrent visit from 2005. Data from 2011 were used only for finding recurrent infections.

A number of sociodemographic and patient-level factors were investigated in the model. Sociodemographic factors included age, gender, race, health insurance type, household income, geographical location, degree of urbanization, and visit year. Age was divided into four categories: $<15,15-44$, $45-64$, and $>64$ years. Household income was represented by the quartile of the median household income in the patient's zip code. Geographical location was defined as southern and northern California, divided near the $37^{\circ}$ latitude (as per the 2010 county-level census data). Degree of urbanization was divided into four categories using the 2003 urban influence codes: large metropolitan area $(\geqslant 1$ million residents); small metropolitan area $(<1$ million 
Table 1. Types of skin and soft tissue infections

\begin{tabular}{llll}
\hline \hline Infection type & ICD-9 code & No. of infections $(\%)$ & $\geqslant 1$ Recurrence \\
\hline Carbuncle and furuncle & $680 . \mathrm{XX}$ & $1097(0 \cdot 56)$ & $257(0 \cdot 80)$ \\
Cellulitis and abscess of finger and toe & $681 . \mathrm{XX}$ & $11669(5 \cdot 91)$ & $1477(4 \cdot 60)$ \\
Impetigo & $684 . \mathrm{XX}$ & $637(0 \cdot 32)$ & $151(0 \cdot 47)$ \\
Other cellulitis and abscess & $682 . \mathrm{XX}$ & $179293(90 \cdot 84)$ & $29077(90 \cdot 59)$ \\
Other local infections of skin and subcutaneous tissue & $686 . \mathrm{XX}$ & $2912(1 \cdot 48)$ & $672(2 \cdot 09)$ \\
Inflammatory disease of breast & $611 \cdot 0$ & $712(0 \cdot 36)$ & $185(0 \cdot 58)$ \\
Other specified diseases of hair and hair follicles & $704 \cdot 8$ & $963(0 \cdot 49)$ & $259(0 \cdot 81)$ \\
Erysipelas & 35 & $88(0 \cdot 04)$ & $20(0 \cdot 06)$ \\
Total & & 197371 & $32098(16 \cdot 26)$ \\
\hline \hline
\end{tabular}

Table 2. Skin and soft tissue infection recurrence by year

\begin{tabular}{llll}
\hline \hline Year & No recurrence & $\geqslant 1$ Recurrence & $>1$ Recurrence \\
\hline 2006 & 32068 & 6394 & 2141 \\
2007 & 31933 & 6108 & 2000 \\
2008 & 33153 & 6362 & 2015 \\
2009 & 33298 & 6467 & 2103 \\
2010 & 34821 & 6767 & 2160 \\
Total & 165273 & 32098 & 10419 \\
\hline \hline
\end{tabular}

residents); micropolitan area; and neither metropolitan nor micropolitan area [24].

Patient-level factors included patient discharge disposition (whether the patient was admitted to the hospital after their ED visit), comorbidities, SSTI treatment factors, including antibiotic injection and incision or drainage, and aspiration. Comorbidities investigated in the model included AIDS; alcohol abuse, drug abuse, and liver cancer; breast cancer, lymph cancer, and solid tumour; chronic pulmonary disorder; diabetes; obesity; and peripheral vascular disorder. In addition, a Charlson comorbidity score [25] was calculated for each patient.

\section{RESULTS}

Of 197371 SSTI patients included in this analysis, $32098(16 \cdot 3 \%)$ had at least one recurrent SSTI visit and $10419(5 \cdot 3 \%)$ had more than one recurrent SSTI visit (Table 2). SSTI recurrent visit rate varied by year, with the highest recurrent visit rate in 2006 (6394 patients, 16.6\%) and the lowest in 2007 (6108 patients, $16 \cdot 1 \%$ ). The overall number of SSTI patients increased slightly over this period from 38462 patients in 2006 to 41588 patients in 2010 (Table 2). The most common diagnosis was cellulitis, which accounted for $96 \cdot 75 \%$ of visits (ICD-9 codes 681 and 682 ), although recurrence rates did not differ for different diagnoses (Table 1).

The demographic predictors included in this analysis were all significantly associated with a patient's odds of having a recurrent ED visit (Table 3). Non-senior adults were more likely than children to have a recurrent SSTI visit [age 18-44 years: odds ratio (OR) $1 \cdot 36,95 \%$ confidence interval (CI) $1 \cdot 27$ $1 \cdot 45$; age $45-64$ years: OR $1 \cdot 19,95 \%$ CI $1 \cdot 11-1 \cdot 27$ ), while the elderly were less likely to have a recurrent visit (OR 0.78, 95\% CI 0.72-0.84). Female patients had $11 \%$ higher odds of a recurrent visit compared to male patients (OR $1 \cdot 11,95 \%$ CI $1 \cdot 08-1 \cdot 14$ ), and non-Hispanic white patients also had significantly higher rates of recurrent visits than other races/ethnicities (Black: OR 0.87, 95\% CI 0.83-0.91; Hispanic: OR 0.90 , 95\% CI 0.87-0.93; Asian or Pacific Islander: OR $0 \cdot 61,95 \%$ CI $0 \cdot 55-0 \cdot 67$ ).

Insurance status was significantly associated with a patient's odds of a recurrent visit. However, while Medicare coverage or self-pay status were both slightly higher compared to patients with private insurance coverage (Medicare: OR 1.09, 95\% CI 1.04-1.13; self-pay: OR $1 \cdot 07,95 \%$ CI $1 \cdot 03-1 \cdot 13$ ), patients with Medicaid were less likely to have a recurrent visit (OR 0.67, 95\% CI 0.64-0·70). Geography and income were also important. Patients from wealthier areas were less likely to have recurrent visits (wealthiest $v s$. poorest quartile: OR $0.92,95 \% \mathrm{CI}$ 0.88-0.96). Patients treated in northern California were slightly more likely to have a recurrent visit compared to patients in southern California (OR 1.03, 95\% CI 1.01-1.06). In addition, patients treated in rural areas (neither metropolitan nor micropolitan) had $22 \%$ lower odds of a recurrent visit compared to 
Table 3. Predictors of skin and soft tissue infection recurrence

\begin{tabular}{|c|c|c|c|}
\hline & $\begin{array}{l}\text { No recurrence } \\
n(\%)\end{array}$ & $\begin{array}{l}\geqslant 1 \text { Recurrence } \\
n(\%)\end{array}$ & OR $(95 \% \mathrm{CI})$ \\
\hline Total & 165273 & 32098 & 197371 \\
\hline \multicolumn{4}{|l|}{ Age, years } \\
\hline$<18$ & $8512(5 \cdot 2)$ & $1207(3 \cdot 8)$ & Ref. \\
\hline $18-44$ & $55415(33 \cdot 5)$ & $13083(40 \cdot 8)$ & $1.36(1.27-1.45)^{*}$ \\
\hline $45-64$ & $66101(40 \cdot 0)$ & $13603(42 \cdot 4)$ & $1 \cdot 19(1 \cdot 11-1 \cdot 27)^{*}$ \\
\hline$>64$ & $35245(21 \cdot 3)$ & $4205(13 \cdot 1)$ & $0.78(0.72-0.84)^{*}$ \\
\hline \multicolumn{4}{|l|}{ Gender } \\
\hline Male & $94128(57 \cdot 0)$ & $17829(55 \cdot 5)$ & Ref. \\
\hline Female & $71145(43 \cdot 0)$ & $14269(44 \cdot 5)$ & $1 \cdot 11(1 \cdot 08-1 \cdot 14) *$ \\
\hline \multicolumn{4}{|l|}{ Race } \\
\hline White & $99186(60 \cdot 0)$ & $19711(61 \cdot 4)$ & Ref. \\
\hline Black & $14259(8 \cdot 6)$ & $2960(9 \cdot 2)$ & $0 \cdot 87(0 \cdot 83-0.91)^{*}$ \\
\hline Hispanic & $43365(26 \cdot 2)$ & $8269(25 \cdot 8)$ & $0.90(0 \cdot 87-0.93)^{*}$ \\
\hline Asian or Pacific Islander & $4988(3 \cdot 0)$ & $436(1 \cdot 4)$ & $0.61(0.55-0.67)^{*}$ \\
\hline Native American & $348(0 \cdot 2)$ & $109(0 \cdot 3)$ & $1 \cdot 11(0 \cdot 89-1 \cdot 39)$ \\
\hline Other & $3127(1.9)$ & $613(1.9)$ & $0.89(0.81-0.97)^{*}$ \\
\hline \multicolumn{4}{|l|}{ Insurance type } \\
\hline Private & $45389(27 \cdot 5)$ & $7449(23 \cdot 2)$ & Ref. \\
\hline Medicare & $36007(21 \cdot 8)$ & $8983(28 \cdot 0)$ & $1.09(1 \cdot 04-1 \cdot 14)^{*}$ \\
\hline Medicaid & $42759(25.9)$ & $5463(17 \cdot 0)$ & $0.67(0.64-0.70) *$ \\
\hline Self-pay & $24668(14 \cdot 9)$ & $6680(20 \cdot 8)$ & $1.07(1.03-1 \cdot 13) *$ \\
\hline Other & $16450(10 \cdot 0)$ & $3523(11 \cdot 0)$ & $1.09(1.03-1 \cdot 15)^{*}$ \\
\hline \multicolumn{4}{|l|}{ Median household income quartile } \\
\hline First (poorest) & $54016(32 \cdot 7)$ & $11925(37 \cdot 2)$ & Ref. \\
\hline Second & $44210(26 \cdot 7)$ & $8864(27 \cdot 6)$ & $0.97(0.94-1.00) *$ \\
\hline Third & $38097(23 \cdot 1)$ & $6744(21 \cdot 0)$ & $0.91(0.88-0.95)^{*}$ \\
\hline Fourth (wealthiest) & $28950(17 \cdot 5)$ & $4565(14 \cdot 2)$ & $0.92(0 \cdot 88-0.96)^{*}$ \\
\hline \multicolumn{4}{|l|}{ Disposition } \\
\hline Routine & $141861(85 \cdot 8)$ & $28777(89 \cdot 7)$ & Ref. \\
\hline Home healthcare & $18816(11 \cdot 4)$ & $2220(6 \cdot 9)$ & $1 \cdot 07(1 \cdot 02-1 \cdot 13)^{*}$ \\
\hline Against medical advice & $4350(2 \cdot 6)$ & $1075(3 \cdot 3)$ & $1 \cdot 16(1 \cdot 09-1 \cdot 25)^{*}$ \\
\hline Discharge alive, destination unknown & $246(0 \cdot 1)$ & $26(0 \cdot 1)$ & $0.86(0 \cdot 57-1 \cdot 30)$ \\
\hline \multicolumn{4}{|l|}{ Region } \\
\hline South & $92969(56 \cdot 3)$ & $17130(53 \cdot 4)$ & Ref. \\
\hline North & $72304(43 \cdot 7)$ & $14968(46 \cdot 6)$ & $1.03(1.01-1.06) *$ \\
\hline \multicolumn{4}{|l|}{ Degree of urbanization } \\
\hline Large metropolitan areas & $119073(72 \cdot 0)$ & $22045(68 \cdot 7)$ & Ref. \\
\hline Small metropolitan areas & $40496(24 \cdot 5)$ & $8773(27 \cdot 3)$ & $0 \cdot 98(0 \cdot 95-1 \cdot 01)$ \\
\hline Micropolitan areas & $4279(2 \cdot 6)$ & $1033(3 \cdot 2)$ & $0 \cdot 94(0 \cdot 87-1 \cdot 01)$ \\
\hline Not metropolitan or micropolitan area & $1425(0 \cdot 9)$ & $247(0 \cdot 8)$ & $0.78(0.68-0.90) *$ \\
\hline \multicolumn{4}{|l|}{ Year } \\
\hline 2006 & $32068(19 \cdot 4)$ & $6394(19 \cdot 9)$ & Ref. \\
\hline 2007 & $31933(19 \cdot 3)$ & $6108(19 \cdot 0)$ & $0.97(0 \cdot 93-1 \cdot 01)$ \\
\hline 2008 & $33153(20 \cdot 1)$ & $6362(19 \cdot 8)$ & $0 \cdot 98(0 \cdot 95-1 \cdot 02)$ \\
\hline 2009 & $33298(20 \cdot 1)$ & $6467(20 \cdot 1)$ & $1 \cdot 01(0.97-1 \cdot 05)$ \\
\hline 2010 & $34821(21 \cdot 1)$ & $6767(21 \cdot 1)$ & $1 \cdot 03(0 \cdot 99-1 \cdot 07)$ \\
\hline \multicolumn{4}{|l|}{ Admitted } \\
\hline No & $58992(35 \cdot 7)$ & $19453(60 \cdot 6)$ & Ref. \\
\hline Yes & $106281(64 \cdot 3)$ & $12645(39 \cdot 4)$ & $0.35(0.34-0.36)^{*}$ \\
\hline \multicolumn{4}{|l|}{ Comorbidities (all binary) } \\
\hline AIDS & $1101(0 \cdot 7)$ & $243(0 \cdot 8)$ & $0.81(0.70-0.94)^{*}$ \\
\hline Abuse (alcohol, drug, liver disease) & $21819(13 \cdot 2)$ & $5497(17 \cdot 1)$ & $1.35(1.32-1.39) *$ \\
\hline Cancer (breast, lymph, solid tumour) & $9901(6 \cdot 0)$ & $1264(3 \cdot 9)$ & $0.87(0.82-0.92)^{*}$ \\
\hline Chronic pulmonary disorder & $17538(10 \cdot 6)$ & $2946(9 \cdot 2)$ & $1 \cdot 03(0 \cdot 98-1 \cdot 08)$ \\
\hline Diabetes & $44516(26 \cdot 9)$ & $7477(23 \cdot 3)$ & $0.92(0.89-0.95)^{*}$ \\
\hline
\end{tabular}


Table 3 (cont.)

\begin{tabular}{llll}
\hline \hline & $\begin{array}{l}\text { No recurrence } \\
n(\%)\end{array}$ & $\begin{array}{l}\geqslant 1 \text { Recurrence } \\
n(\%)\end{array}$ & OR $(95 \% \mathrm{CI})$ \\
\hline $\begin{array}{l}\text { Obesity } \\
\text { Peripheral vascular disorder }\end{array}$ & $19088(11 \cdot 5)$ & $3048(9 \cdot 5)$ & $1 \cdot 30(1 \cdot 24-1 \cdot 36)^{*}$ \\
Procedures & $5557(3 \cdot 4)$ & $729(2 \cdot 3)$ & $1 \cdot 03(0 \cdot 95-1 \cdot 12)$ \\
$\quad$ Antibiotic injection & $2791(1 \cdot 7)$ & $341(1 \cdot 1)$ & $1 \cdot 05(0 \cdot 94-1 \cdot 18)$ \\
$\quad$ Incision/drain and aspiration & $39743(24 \cdot 0)$ & $8489(26 \cdot 4)$ & $1 \cdot 10(1 \cdot 07-1 \cdot 13)^{*}$ \\
Charlson score (mean) & $2 \cdot 37$ & $2 \cdot 77$ & $1 \cdot 07(1 \cdot 07-1 \cdot 08)^{*}$ \\
\hline \hline
\end{tabular}

OR, Odds ratio; CI, confidence interval.

* Significant at the 0.05 level.

patients living in metropolitan areas (OR 0.78, 95\% CI 0.68-0.90).

A patient's health condition was significantly associated with the odds of recurrent visits. Admitted patients, who are likely sicker than discharged patients, had $65 \%$ lower odds of a recurrent visit compared to patients who were not admitted (OR 0.35, $95 \%$ CI $0 \cdot 34-0 \cdot 36$ ), however, those who were discharged to home healthcare had higher odds of recurrence (OR 1.07, 95\% CI 1.07-1·08). Patients' odds of a recurrent visit increased by $7 \%$ with each 1-unit increase in Charlson comorbidity score (OR 1.07, $95 \%$ CI $1 \cdot 07,1 \cdot 08)$. However, not all of the individual comorbidities analysed were associated with higher odds of a recurrent visit. For instance, patients with a history of drug or alcohol abuse or liver disease had a higher odds of a recurrent visit (OR 1.35, 95\% CI 1.32-1.39). Similarly, obese patients had 30\% higher odds of a recurrent visit (OR 1.30, 95\% CI 1.24-1.36). However, patients with AIDS, cancer, and diabetes all had lower odds of recurrent visits compared to patients without these conditions (AIDS: OR $0 \cdot 81,95 \%$ CI $0 \cdot 70-0.94$ ); cancer: OR $0 \cdot 87,95 \%$ CI $0 \cdot 82-0 \cdot 92$; diabetes: OR $0 \cdot 92,95 \%$ CI $0 \cdot 89-0 \cdot 95)$. Moreover, certain comorbidities, such as chronic pulmonary disorder and peripheral vascular disorder were not associated with a patient's likelihood of an SSTI recurrent visit (Table 3).

Treatment (or lack thereof) also impacted the likelihood of a recurrent visit. Patients whose infections were drained or aspirated had higher odds of a recurrent visit compared to patients who did not receive these treatments (OR $1 \cdot 10,95 \%$ CI $1 \cdot 07-1 \cdot 13$ ). Patients who were discharged against medical advice were also more likely to have an SSTI recurrent visit (OR $1 \cdot 16,95 \%$ CI 1·09-1·25). No significant difference in the odds of an SSTI recurrent visit between the years included in this analysis was found, and a proportional trend analysis also showed no significant trend in recurrent visits over time $\left(\chi^{2}\right.$ trend $=0.68, P=$ 0.409).

Many of the predictors included in this analysis were also significantly associated with a patient's odds of having more than one recurrent visit (Supplementary Table S1). In general, the magnitude of association was greater when predicting multiple recurrent visits than one or more recurrent visits. For example, adults aged $18-44$ years had $41 \%$ higher odds of having multiple recurrent visits compared to patients asged $<18$ years (OR 1.41, 95\% CI 1.26 1.57 ), in contrast to $36 \%$ higher odds of having any recurrent visit (OR 1.36, 95\% CI 1.27-1.45). However, some factors that were associated with the odds of having one or more recurrent visit were not significantly associated with the odds of having multiple recurrent visits. Household income, patient's disposition, and geographical location were not predictive of a patient's odds of having multiple recurrent visits, though these factors were significantly associated with odds of recurrent visits in general (Table 3).

Finally, although the logistic regression shows no significant difference in odds of recurrent visits between years, a proportional trend analysis showed a very slight but significant decreasing trend in multiple recurrent visits over time (slope $=-0.0007, \chi^{2}$ trend $=3.94, P=0.047$ ).

\section{DISCUSSION}

To our knowledge, this study is the first to evaluate rates and sociodemographic and clinical predictors of visits for recurrent SSTI in the ED at such a large scale. We found that recurrent visits are a common problem, as over $16 \%$ of all SSTI patients returned to the ED at least one time for a similar condition 
in the 6 months following an initial SSTI. While these rates are significantly lower than the $28-39 \%$ recurrence rate of prior studies [15, 16], prior studies included only patients with purulent wounds that could be cultured. However, as discussed below, our study examines only those patients that presented to the ED that subsequently sought care for their recurrent SSTI in the ED. Thus our estimates may be lower than true recurrence rates as some patients may have sought care for recurrent infections in non-ED settings such as clinics or with their primary-care provider.

SSTIs are disproportionately seen in EDs. In this large sample, we found multiple sociodemographic factors associated with recurrent ED visits for SSTIs, including age, race/ethnicity, and insurance status. The most surprising of these was race, as previous studies have not identified race-associated differences in recurrence rate $[15,26]$. However, it is likely that the observed difference is related to differences in healthcare utilization patterns, and not differences in predisposition to recurrent SSTIs. Disparities in healthcare utilization and outcomes could potentially be addressed with ED-based interventions to improve appropriate treatment to decrease risk recurrence, such as education on home-based management and hygiene, as well as linkages to appropriate care.

Interestingly, while patients seeking care in the ED generally are more likely to be underinsured and of minority status, we found that minority patients and those with Medicaid status were less likely to have a recurrent SSTI visit, whereas lower household income was associated with increased likelihood of a recurrent visit. This conflicting result may perhaps be explained by the higher rate of recurrent visits among self-pay patients (typically a poorer group). Together these findings suggest that having Medicaid may provide access to additional resources (e.g. primary-care providers) compared to those with no insurance that prevents ED recidivism. Further investigation of socioeconomics and SSTI recurrence is warranted.

We found that certain comorbidities, specifically obesity, drug or alcohol abuse, and liver disease were associated with increased likelihood of recurrent SSTI visits. While CA-MRSA, a primary cause of SSTIs [1-4], can strike healthy individuals, common comorbidities may be important predictors of community-acquired $S$. aureus infection [11, 27]. Our findings suggest these populations are not only at higher risk of SSTIs but of SSTI recurrences as well.

Recent data on SSTIs in more than 2 million people found that diabetics have a fivefold increased SSTI incidence $(4 \cdot 9 \%$ vs. $0 \cdot 8 \%$ per year) as well as higher rates of SSTI complications, including hospitalization, bacteraemia, and sepsis [28]. Recent data also suggest obesity is a risk factor for CA-MRSA, including recurrent invasive disease [27]. Interestingly, we found that diabetes was associated with a lower risk of recurrent SSTIs, as were AIDS and cancer. The reasons for this are unclear, but may stem from the fact that these populations have lower barriers to access care and perhaps present in an earlier stage of infection that is less likely to relapse or are perhaps treated more aggressively than non-diabetic patients. Hospitalized patients had decreased odds of recurrent visits, which may reflect a more thorough evaluation and aggressive medical and surgical treatment before being released or perhaps access to post-discharge care that patients who are not hospitalized lack (see Supplementary Tables S2 and S3).

Most studies of MRSA-related SSTIs have been performed in urban areas, where the epidemic was first described [3]. Data on the incidence of SSTIs or CA-MRSA in non-urban or suburban regions other than Native American communities are scarce [29, 30]. In this study, patients in low-density or rural areas of California had significantly reduced odds of recurrent visits. Reasons for this are unclear and perhaps related to rural areas sought care for their infections with primary-care providers. Alternately, prior studies have demonstrated that household crowding is associated with CA-MRSA infection and recurrence [31] and it is possible that persons living in areas of low density may experience less household crowding, although they face increased barriers to access which may reduce their recurrent visit rate [32].

Hospitalization for patients diagnosed with cutaneous abscess in the ED is uncommon, with fewer than $5 \%$ requiring admission [26]. In terms of treatment, while we lacked much clinical detail, patients with drained or aspirated SSTIs had higher odds of a recurrent visit compared to patients who did not receive these treatments. Although we were unable to confirm a specific diagnosis of cellulitis versus abscess in these patients due to coding vagaries (see below), the higher odds of recurrent visits associated with these procedures may reflect that cutaneous abscess, which are associated with CA-MRSA [6, 7], have higher rates of recurrence compared to nonsuppurative cellulitis.

As noted above, one major limitation of our investigation is that the databases that we used allowed us only to examine SSTI recurrences that represent to 
EDs. Thus we cannot truly examine recurrence rates in this population and instead are focused on ED recidivism, which likely significantly underestimates recurrent disease. Additionally, our design may introduce bias in our analysis as some subpopulations may be more likely to receive care outside EDs for recurrent infection. Nevertheless, EDs remain a primary source of care for a large number of patients with SSTIs $[6,7]$ and it may be unlikely that a single ED visit for an SSTI would change a patients access or preferred point of care for a semi-urgent issue. Another limitation is our use of an administrative datasets to try to categorize of SSTI subtypes; more specifically, the ICD-9 billing code for abscess and cellulitis is the same (682.XX) and administrative databases cannot untangle these two forms of SSTI and these infection subtypes likely have different aetiologies and prognoses [33]. Cutaneous abscesses may be more likely to recur than other types of SSTIs, possibly due to their association with CA-MRSA [16] the most common bacterial cause for culturable SSTIs nationwide [1-4]. While we were not able to assess MRSA as an aetiology of SSTI due to the lack of microbiology results in these datasets, our assumption that cutaneous abscesses are driving the higher recurrence rate is supported by the higher odds of a recurrent ED visit in adults aged 15-44 years, since CA-MRSA abscesses are more likely to occur in this population [3]. However, secular S. aureus epidemiological trends, which may have peaked in the mid-2000s and subsequently decreased nationwide, may also be driving some of the results [34, 35].

There are additional limitations to our study. Our analysis did not account for patients that moved out of the state, came from out of the state, or died. Thus, some of the associations identified in this analysis may be more indicative of patients who are likely to seek follow-up care at an ED rather than those who are likely to have recurrent SSTIs. Additionally, reliance on ICD-9 codes may not have captured all patients presenting to the ED for an SSTI, as we only included patients with a primary diagnosis that met our criteria. The finding that admitted patients were significantly less likely to have a recurrent visit for SSTI leads to concerns that more critically ill patients with sepsis as their primary diagnosis may have been excluded.

Finally, SEDD is an administrative dataset and information on potentially important clinical variables, behavioral variables, and treatment is limited or non-existent. Given literature that suggests antibiotic treatment may be associated with decreased recurrent lesions and improved initial outcomes [36, 37], we were unable to evaluate the role of antibiotic treatment in recurrent visits. The lower threshold for antibiotic treatment in immunosuppressed patients is also likely an important confounder. Additionally combining various categories of SSTIs (e.g. cellulitis, abscess, furunculosis), may be conceptually limiting in our analysis. Nevertheless, while we were unable to verify the diagnosis of abscess, the presence of aspiration or incision and drainage procedures may serve as a proxy for cutaneous abscess. We were also unable to confirm that subsequent ED visits for SSTIs were not associated with treatment failure for the initial SSTI rather than a true recurrence, especially given patients will often return to the ED for re-evaluation per standard ED practice. However, the exclusion of visits within 2 weeks of the initial visit likely diminishes this likelihood.

In conclusion, we found multiple sociodemographic factors and comorbidities associated with returning to the ED for a recurrent SSTI in a large population of patients in California. Identification of patients at risk for recurrent ED visits for SSTIs could help target therapy such as improving care access, or perhaps treating high risk patients with more appropriate antibiotics. Further investigation should better define the characteristics of populations at risk for recurrent SSTI visits, including their infection subtype, as those at risk for recurrent infection could be targets for interventions that could reduce the burden of repeat visits for this very common condition.

\section{SUPPLEMENTARY MATERIAL}

For supplementary material accompanying this paper visit https://doi.org/10.1017/S0950268816002855.

\section{ACKNOWLEDGEMENTS}

This research received no specific grant from any funding agency, commercial or not-for-profit sectors.

\section{DECLARATION OF INTEREST}

None.

\section{REFERENCES}

1. Frazee BW, et al. High prevalence of methicillinresistant Staphylococcus aureus in emergency department skin and soft tissue infections. Annals of Emergency Medicine 2005; 45: 311-320. 
2. Hawkes M, et al. Community-associated MRSA: superbug at our doorstep. Canadian Medical Association Journal 2007; 176: 54-56.

3. Moran GJ, et al. Methicillin-resistant S. aureus infections among patients in the emergency department. New England Journal of Medicine 2006; 355: 666-674.

4. Zetola N, et al. Community-acquired meticillin-resistant Staphylococcus aureus: an emerging threat. Lancet Infectious Dseases 2005; 5: 275-286.

5. Miller LG, et al. Incidence of skin and soft tissue infections in ambulatory and inpatient settings, 2005-2010. BMC Infectious Diseases 2015; 15: 1.

6. Taira BR, et al. National epidemiology of cutaneous abscesses: 1996 to 2005. American Journal of Emergency Medicine 2009; 27: 289-292.

7. Pallin DJ, et al. Increased US emergency department visits for skin and soft tissue infections, and changes in antibiotic choices, during the emergence of communityassociated methicillin-resistant Staphylococcus aureus. Annals of Emergency Medicine 2008; 51: 291-298.

8. May L, Mullins P, Pines J. Demographic and treatment patterns for infections in ambulatory settings in the United States, 2006-2010. Academic Emergency Medicine 2014; 21: 17-24.

9. Graber CJ, et al. Recurrence of skin and soft tissue infection caused by methicillin-resistant Staphylococcus aureus in a HIV primary care clinic. Journal of Acquired Immune Deficiency Syndromes 2008; 49: 231-233.

10. Shastry L, Rahimian J, Lascher S. Communityassociated methicillin-resistant Staphylococcus aureus skin and soft tissue infections in men who have sex with men in New York City. Archives of Internal Medicine 2007; 167: 854-857.

11. David MZ, Daum RS. Community-associated methicillin-resistant Staphylococcus aureus: epidemiology and clinical consequences of an emerging epidemic. Clinical Microbiology Reviews 2010; 23: 616-687.

12. Crum-Cianflone N, Weekes J, Bavaro M. Recurrent community-associated methicillin-resistant Staphylococcus aureus infections among HIV-infected persons: incidence and risk factors. AIDS Patient Care and STDS 2009; 23: 499-502.

13. Sreeramoju P, et al. Recurrent skin and soft tissue infections due to methicillin-resistant Staphylococcus aureus requiring operative debridement. American Journal of Surgery 2011; 201: 216-220.

14. David MZ, et al. Predominance of methicillin-resistant Staphylococcus aureus among pathogens causing skin and soft tissue infections in a large urban jail: risk factors and recurrence rates. Journal of Clinical Microbiology 2008; 46: 3222-3227.

15. Miller LG, et al. Staphylococcus aureus skin infection recurrences among household members: an examination of host, behavioral, and pathogen-level predictors. Clinical Infectious Diseases 2015; 60: 753-763.

16. May LS, et al. Treatment failure outcomes for emergency department patients with skin and soft tissue infections. Western Journal of Emergency Medicine 2015; 16: 642.

17. U.S. Census Bureau. 2010 Census (http://www.census. gov/2010census/). Accessed 16 March 2016.
18. U.S. Census Bureau. California QuickFacts from the US Census Bureau 2010 (http://www.census.gov/quickfacts/ table/PST045215/06). Accessed 16 March 2016.

19. Hing E, Rui P. Emergency department use in the country's five most populous states and the total United States, 2012. NCHS Data Brief 2016; 000: 1-8.

20. Centers for Disease Control. Methicillin-resistant Staphylococcus aureus infections in correctional facilities-Georgia, California, and Texas, 2001-2003. Morbidity and Mortality Weekly Report 2003; 52: 992.

21. Diep BA, et al. Widespread skin and soft-tissue infections due to two methicillin-resistant Staphylococcus aureus strains harboring the genes for Panton-Valentine leucocidin. Journal of Clinical Microbiology 2004; 42: 2080-2084.

22. Pan ES, et al. Increasing prevalence of methicillinresistant Staphylococcus aureus infection in California jails. Clinical Infectious Diseases 2003; 37: 1384-1388.

23. Miller LGD, et al. Recurrence or internal infections after treatment of uncomplicated skin and soft tissue infection (USSTI) among patients enrolled in DMID protocol 07-0051 [a multi-center randomized double blind controlled trial of clindamycin (CLINDA) versus trimethoprim-sulfamethoxazole (TMP-SMX) SSTI]. IDWeek, 2013, Abstract 625.

24. United States Department of Agriculture Economic Research Service. The 2013 urban influence codes 2013 (http://www.ers.usda.gov/data-products/urban-influencecodes.aspx). Accessed 16 March 2016.

25. Charlson ME, et al. A new method of classifying prognostic comorbidity in longitudinal studies: development and validation. Journal of Chronic Diseases 1987; 40: 373-383.

26. May LS, et al. A randomized clinical trial comparing use of rapid molecular testing for Staphylococcus aureus for patients with cutaneous abscesses in the emergency department with standard of care. Infection Control \& Hospital Epidemiology 2015; 36: 1423-1430.

27. Thana Khawcharoenporn $\mathbf{M}$, et al. Risk factors for community-associated methicillin-resistant Staphylococcus aureus cellulitis - and the value of recognition. Hawai' $i$ Medical 2010; 69: 232.

28. Suaya JA, et al. Skin and soft tissue infections and associated complications among commercially insured patients aged 0-64 years with and without diabetes in the US. PLoS ONE 2013; 8: e60057.

29. David MZ, et al. Molecular epidemiology of methicillinresistant Staphylococcus aureus, rural southwestern Alaska. Emerging Infectious Diseases 2008; 14: 1693 1699.

30. Stemper ME, Shukla SK, Reed KD. Emergence and spread of community-associated methicillin-resistant Staphylococcus aureus in rural Wisconsin, 1989 to 1999. Journal of Clinical Microbiology 2004; 42: 5673-80.

31. Golding GR, et al. A comparison of risk factors associated with community-associated methicillin-resistant and -susceptible Staphylococcus aureus infections in remote communities. Epidemiology and Infection 2010; 138 (Special Issue 05): 730-737. 
32. Casey MM, Thiede Call K, Klingner JM. Are rural residents less likely to obtain recommended preventive healthcare services? American Journal of Preventive Medicine 2001; 21: 182-188.

33. Stevens DL, et al. Practice guidelines for the diagnosis and management of skin and soft tissue infections: 2014 update by the infectious diseases society of America. Clinical Infectious Diseases 2014; 59: 147-59.

34. Moran GJ, et al. Methicillin-resistant Staphylococcus aureus in community-acquired skin infections. Emerging Infectious Diseases 2005; 11: 928-930.
35. Delorenze GN, et al. Trends in annual incidence of methicillin-resistant Staphylococcus aureus (MRSA) infection in HIV-infected and HIV-uninfected patients. Epidemiology and Infection 2013; 141: 2392-2402.

36. Talan DA, et al. A randomized trial of clindamycin versus trimethoprim-sulfamethoxazole for uncomplicated wound infection. Clinical Infectious Diseases 2016; 62: 1505-1513.

37. Talan DA, et al. Trimethoprim-sulfamethoxazole versus placebo for uncomplicated skin abscess. New England Journal of Medicine 2016; 374: 823-832. 